\title{
Cosmic Ray Anisotropy and Solar Activity
}

\author{
Rajesh K. Mishra ${ }^{1}$ and Rekha Agarwal Mishra ${ }^{2}$ \\ ${ }^{1}$ Computer and Information Technology Section, Tropical Forest Research Institute, \\ P.O.: RFRC, Mandla Road, Jabalpur (M.P.) 482 021, India \\ ${ }^{2}$ Department of Physics, Govt. Model Science College (Autonomous), Jabalpur (M.P.) 482 001, India
}

Received on 23 July, 2007

\begin{abstract}
In this paper an attempt is made to study the occurrence of a large number of high amplitude anisotropic wave train events in cosmic ray intensity during 1981-1994 and to identify a possible correlation with solar activity using the hourly neutron monitor data of Deep River station. The diurnal time of maximum for both HAE as well as for all days is found to significantly shift towards an earlier time as compared to the corotational/azimuthal direction since the year 1991 onward. It is found that diurnal amplitude and 10.7-cm solar radio flux significantly deviates and reaches to its maximum and phase remains in the corotational direction during the years close to solar activity maximum for HAE. The occurrence of high amplitude anisotropic wave train events is dominant during solar activity minimum as well as maximum years. The amplitude as well as phase of the cosmic ray diurnal anisotropy during HAEs is well correlated with solar activity. The amplitude as well as phase of diurnal anisotropy shows very nominal correlation with $10.7-\mathrm{cm}$ solar radio flux $(\mathrm{r}=0.48,0.47)$. However, the frequency of occurrence of HAEs shows a very weak correlation $(r=-0.36)$ with $10.7-\mathrm{cm}$ solar radio flux.
\end{abstract}

Keywords: Cosmic ray; Solar cycle; 10.7-cm solar radio flux and anisotropy

\section{INTRODUCTION}

The $\sim 11$-year solar cycle variation in cosmic ray intensity observed at Earth is anti-correlated with solar activity with some time lag. It has been known for a long time that the intensity as well as the energy spectrum of the galactic cosmic rays is modulated by solar activity. It is now well-established fact that there is an inverse correlation between cosmic ray intensity and solar activity $[1,2]$. The details of the cosmic ray modulation and variation of time-lag factor are still a matter of great interest. Many researchers have pointed out the anomalous phenomena in the solar modulation of cosmic rays in addition to variation in time lag for the odd and even cycles [3-6]. Earlier, correlative analysis between the cosmic ray intensity and solar activity parameters like sunspot number, grouped solar flares and geomagnetic activity index Ap have been performed for low and medium cut off rigidity stations [7-9].

A number of high amplitude events have been observed with a significant shift in the diurnal time of maximum to corotational direction or later hours [10 and references therein]. Agrawal and Bercovitch [11] have shown that the direction of the 22-year component is perpendicular to the diurnal anisotropy vector and is along the line $162^{\circ}$ east of the SunEarh line; they have attributed the 11-year component to the variation of cut-off rigidity.

Ahluwalia [12] suggested that the diurnal anisotropy is unidirectional during 1957-1970 having a maximum in the corotation direction $(1800 \mathrm{Hr}$ ) and during 1971-1978 the anisotropy consists of two components, one in the corotation direction $(1800 \mathrm{Hr})$ and the other in the radial $(1200 \mathrm{Hr})$ similar to the concept proposed by Quenby and Hashim [13]. The average diurnal anisotropy vector has been explained as a consequence of the equilibrium established between the radial convection of the cosmic ray particles by solar wind and the inward diffusion of particles along the interplanetary magnetic fields due to the radial gradient $[14,15,16]$; the anisotropy is simply visualized as corotation of particles with the solar system magnetic fields $[17,18]$. Further, a detailed analysis of diurnal anisotropy vectors on a long-term basis [19, 20, 21] and on a day-to-day basis [22] clearly indicates that a corotation theory derived by the convection-diffusion model is insufficient to understand the diurnal anisotropy characteristics and the systematic shifting of the average diurnal anisotropy to earlier hours envisages the need of an additional mechanism for explaining the long-term behaviour of diurnal anisotropy.

Tiwari et al. [23] performed systematic correlative study to establish the relationship of cosmic ray anisotropy with solar activity parameters for low and high cut-off rigidity neutron monitoring stations Kiel and Tokyo. They observed a positive correlation both for amplitude and phase of the diurnal anisotropy with sunspot numbers. They also noticed that the semidiurnal phase is positively correlated with sunspot numbers for both the stations, whereas, semidiurnal amplitude shows small negative correlation with sunspot numbers. Kane [24] reported that the recovery of cosmic ray intensity have followed two distinct patterns, slow recovery during odd solar activity cycles 17, 19,21 and fast recovery during even solar activity cycles 18, 20. Kane [24] noticed that the sunspot number pattern remains different in even cycles as compared to the odd cycles in cycles 17-22, with step functions almost similar for sunspots and cosmic rays in even cycles. According to Kane [24] the differential effects on cosmic rays in alternate cycles could be related to sunspot activity.

The purpose of this work is to investigate the solar activity dependence of the diurnal anisotropy vectors over the period 1981-1994 and to interpret the behaviour of the diurnal anisotropy of the HAE events in terms of the distribution and characteristics of the diurnal vectors. 


\section{DATA ANALYSIS}

The pressure corrected data of Deep River Neutron Monitor NM (data from http://spidr.ngdc.noaa.gov/NeutronMonitor) (cut off rigidity $=1.02 \mathrm{GV}$, Latitude $=46.1^{\circ} \mathrm{N}$, Longitude $=282.5^{\circ} \mathrm{E}$, Altitude $=145 \mathrm{M}$ ) has been subjected to Fourier Analysis for the period 1981-94 after applying the trend correction to have the amplitude (\%) and phase (hr) of the diurnal anisotropy of cosmic ray intensity for unusually high amplitude events, as well as for all days. The amplitude of the diurnal anisotropy on an annual average basis is found to be $\leq 0.4 \%$; hence $0.4 \%$ has been taken as the reference line in order to select high amplitude events.

The standard errors of the harmonic coefficients for long period depends upon the distribution of values of $\mathrm{A}$ and $\mathrm{B}$. Assuming that the hourly values follows a standard Gaussian distribution, the standard deviations are obtained from the individual values of $\mathrm{A}$ and $\mathrm{B}$, by calculating the harmonic coefficients for each day during any period.

The mean value of the A and B are given by

$$
\bar{A}=\frac{1}{N}=\sum_{i=1}^{N} A_{i}
$$

and

$$
\bar{B}=\frac{1}{N}=\sum_{i=1}^{N} B_{i}
$$

The standard deviation of $\mathrm{A}$ and $\mathrm{B}$ are given by

$$
\begin{aligned}
& \sigma_{A}^{2}=\frac{1}{N-1} \sum_{i=1}^{N}\left(A_{i}-\bar{A}\right)^{2} \\
& \sigma_{B}^{2}=\frac{1}{N-1} \sum_{i=1}^{N}\left(B_{i}-\bar{B}\right)^{2}
\end{aligned}
$$

Where $\mathrm{N}$ denotes the number of days used in the analysis. The standard error of mean values of $\mathrm{A}$ and $\mathrm{B}$ is given by

$$
\begin{gathered}
\sigma_{\bar{A}}=\sigma_{A} N^{1 / 2} \\
\sigma_{\bar{B}}=\frac{\sigma_{B}}{N^{1 / 2}}
\end{gathered}
$$
by

The standard errors of amplitude and phase will be given

$$
\begin{aligned}
& \sigma_{R}^{2}=\frac{\left(A^{2} \sigma_{A}^{2}+B^{2} \sigma_{B}^{2}\right)}{\left(A^{2}+B^{2}\right)} \\
& \sigma_{\phi}^{2}=\frac{\left(B^{2} \sigma_{A}^{2}+A^{2} \sigma_{B}^{2}\right)}{\left(A^{2}+B^{2}\right)}
\end{aligned}
$$

If Gaussion distribution is assumed then

$$
\sigma_{A}=\sigma_{B}
$$

and in that case above expression reduces to

$$
\sigma_{R}^{2}=\sigma_{A}^{2}=\sigma_{B}^{2}
$$

and

$$
\sigma_{\phi}^{2}=\sigma_{R}^{2}
$$

The days having abnormally high diurnal amplitude $(>0.4$ $\%$ ) for a successive number of five or more days have been selected as high amplitude anisotropic wave train events. The anisotropic wave train events are identified using the hourly plots of cosmic ray intensity recorded at ground-based neutron monitoring station. As such, we have thirty-seven unusually high amplitude wave train events during the period 1981-1994. The average values of 10.7-cm Solar Radio Flux (SRF) for each corresponding HAE event have been used in the present analysis.

\section{RESULTS AND DISCUSSION}

The Sun has been continuously monitored, by using ground-based detectors, which are still very important measuring tools to study the Sun and it's continuously changing outputs, which significantly modulates the cosmic ray intensity as well as produce geomagnetic disturbances. Previously various indices have been standardized representing various facts of the solar phenomena occurring on the photosphere, chromosphere and corona. These are sunspot numbers, 10.7$\mathrm{cm}$ solar radio flux (or $2800 \mathrm{MHz}$ radio emission), grouped solar flares, solar flare index, sunspot area, grouped sunspot numbers and coronal index. Mostly, all the investigators have generally used the sunspot numbers to represent solar activity for various studies in their investigations associating phenomena between the Sun and the Earth. Later on, with the availability of other solar indices, either the sunspot numbers or $10.7-\mathrm{cm}$ solar radio flux has also been used for solar terrestrial relationship studies. For the present study, the authors have used the daily average of the $10.7-\mathrm{cm}$ solar radio flux to represent solar activity.

The long term variation of the amplitude (\%) and time of maximum (Hr) of diurnal anisotropy for each HAE is plotted for the period 1981-1994 and shown in Fig. 1 along with the corresponding 10.7-cm solar radio flux. It can be clearly seen from the figure that the amplitude of the diurnal anisotropy consistently remains constant $(\sim 0.6 \%)$ during 1981-86. The amplitude then increases sharply and reaches its maximum (1.2\%) during the year 1989. However, the $10.7-\mathrm{cm}$ solar radio flux having its first maximum (274) on 1981 decreases sharply from 1981 to 1986 with some fluctuations during 1984. The 10.7-cm solar radio flux start increasing from 1986 


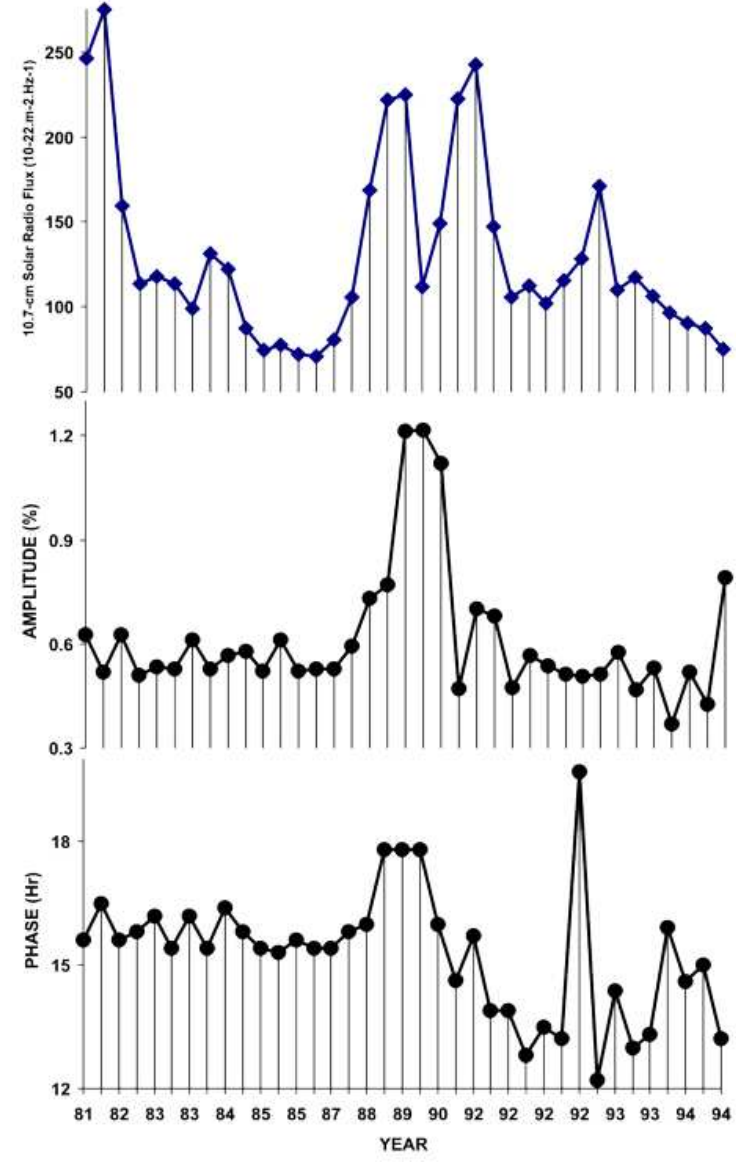

FIG. 1: The long-term variation of observed cosmic-ray diurnal amplitude $(\%)$ and the time of maximum $(\mathrm{Hr})$ for each HAE event for Deep River NM station is shown as a function of solar cycle represented by 10.7-cm solar radio flux for the period 1981-1994.

(solar activity minimum) and reaches its second maximum (225) during 1989. The diurnal amplitude and 10.7-cm solar radio flux both start decreasing from 1989 and the diurnal amplitude reaches to its minimum on 1990 and then found to remain statistically constant $(\sim 0.6 \%)$ onwards. However, the $10.7-\mathrm{cm}$ solar radio flux reaches its minimum on 1990, then increases sharply and reaches its third maximum during 1992. It is also evident from the figure that the amplitude distribution shows some peaks corresponding to $10.7-\mathrm{cm}$ solar radio flux maximum during the year 1989-90 close to the solar activity maximum years. Further, we find from the figure that the observed diurnal time of maximum remains in the corotational/azimuthal direction $(\approx 15.5 \mathrm{Hr})$ at Deep River station for majority of the events. The direction of the anisotropy observed to shift towards later hours as compared to the corotational/azimuthal $(\approx 15.5 \mathrm{Hr})$ direction at Deep River during the year 1989-90 (close to solar activity maximum years) and for one of the event of 1992. Thus, from the above findings we can say that diurnal amplitude and $10.7-\mathrm{cm}$ solar radio flux significantly deviates and reaches its maximum for majority of the HAE, whereas the phase remains in the corotational direction $(\approx 15.5 \mathrm{Hr})$ at Deep River station. This leads us to conclude that the amplitude as well as phase is well correlated with solar activity cycle during high amplitude days. These observations are found to be partially consistent with that of Kumar et al. [25] and Ananth et al. [26], where they suggested that the amplitude of the diurnal anisotropy is correlated with the solar cycle but the direction of the anisotropy is not correlated with the solar cycle and shows a systematic phase shift to earlier hours.

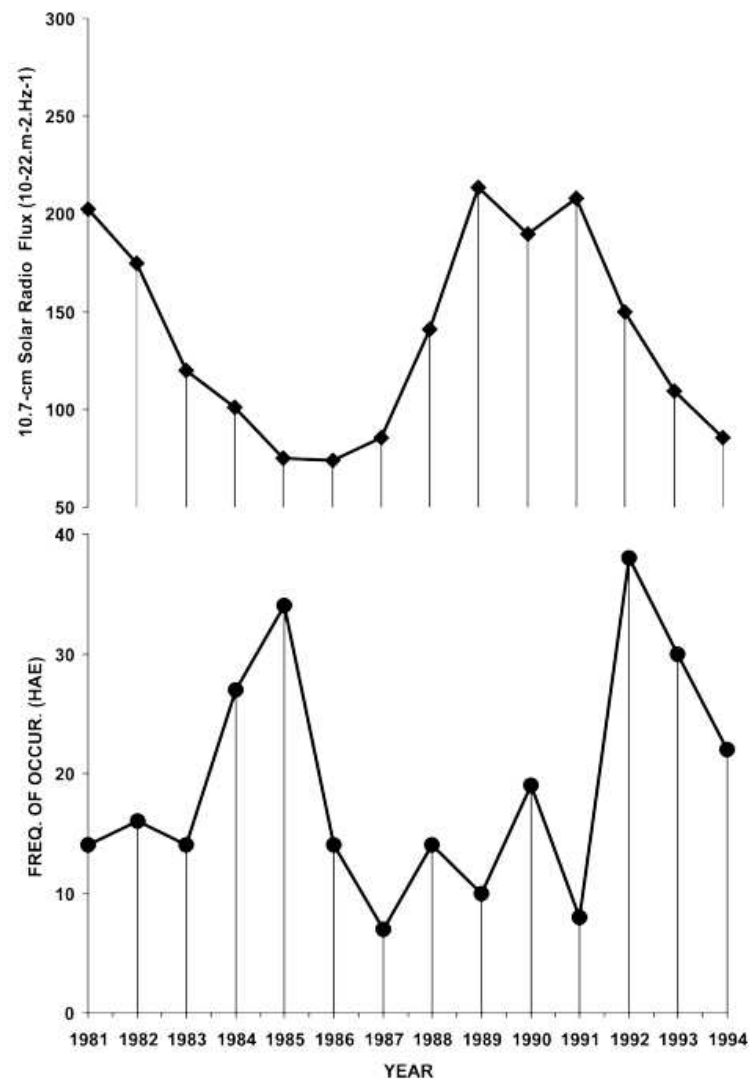

FIG. 2: Annual averages values of first harmonic (a) diurnal amplitude (\%) and (b) diurnal phase (Hr) of Deep River NM station for HAE events and all days (365/366) during the period1981-94.

For comparison purpose, we have also analysed the amplitude and phase for all days (365/366) during 1981-94 and plotted alongwith yearly average for all the HAE's and shown if Fig. 2. The amplitude of the diurnal anisotropy for all days is observed to remain $\sim 0.35 \%$ with some peaks during 1985,1989 and 1994 and dips during 1987 and 1991. The amplitude for HAEs is found to remain $\sim 0.5 \%$ throughout the period. It is also evident from the plot that the amplitude starts increasing from 1987 (solar activity minimum) and reaches its maximum $(>1 \%$ ) during 1990 (solar activity maximum) and then decreases and reaches its usual value in 1991. As depicted, the amplitude significantly remains high for all the HAEs as compared to all days throughout the period (which is expected as we have selected the high amplitude days). On the other hand, the diurnal time of maximum for all days sig- 
nificantly shifts towards a later time as compared to the corotational direction $(\approx 15.5 \mathrm{Hr})$ at Deep River station up to 1991 and then shift towards an earlier time as compared to the corotational direction $(\approx 15.5 \mathrm{Hr})$. The diurnal phase for HAE is observed to remain along the corotational direction $(\approx 15.5$ Hr) up to 1988. The diurnal phase is observed to shifts towards later hours during 1989-90 close to solar activity maximum years and towards earlier hours during 1991-94 as compared to the corotational direction $(\approx 15.5 \mathrm{Hr})$ at Deep River station.
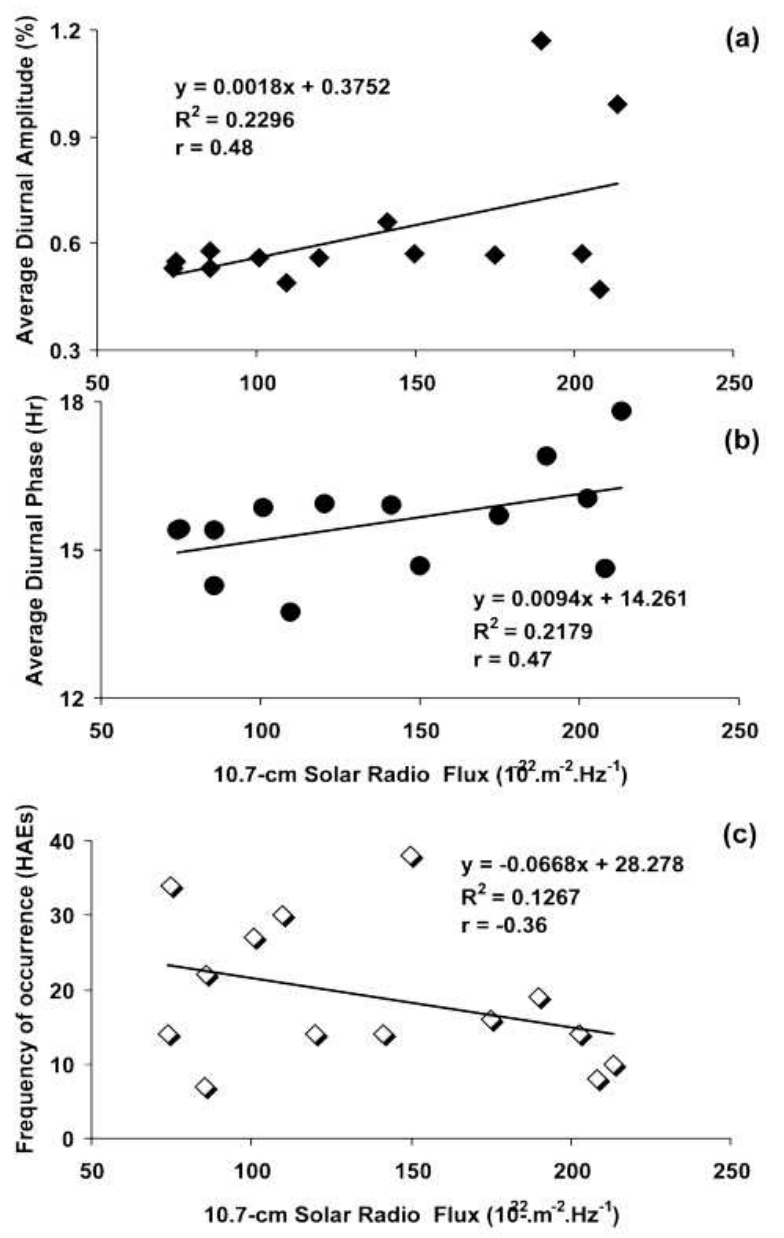

FIG. 3: The frequency of occurrence of days of high amplitude events for Deep River NM station along with annual average 10.7$\mathrm{cm}$ solar radio flux for each year, during the period 1981-1994.

The frequency distribution of high amplitude anisotropic wave train events for each year is plotted in Fig. 3. For comparison purpose, the annual variation in the $10.7-\mathrm{cm}$ solar radio flux indicating the solar activity is also shown in the same figure. The figure clearly illustrates that the distribution of high amplitude days presents a very interesting picture. We observe that the occurrence of high amplitude days is dominant during 1984-85, close to solar activity minimum year and during 1992-93, close to solar activity maximum year showing two peaks during these years. It is noteworthy from the shape of the plot that the occurrence of HAEs found to be anticorrelated with 10.7-cm solar radio flux. These observations are in partial agreement with the results obtained by Kudo and Mori [27] on the 11-year enhancements of diurnal amplitudes. These observations clearly suggest that HAE events do contribute significantly to the long-term variation of amplitude as well as time of maximum of cosmic ray diurnal anisotropy.

(a)

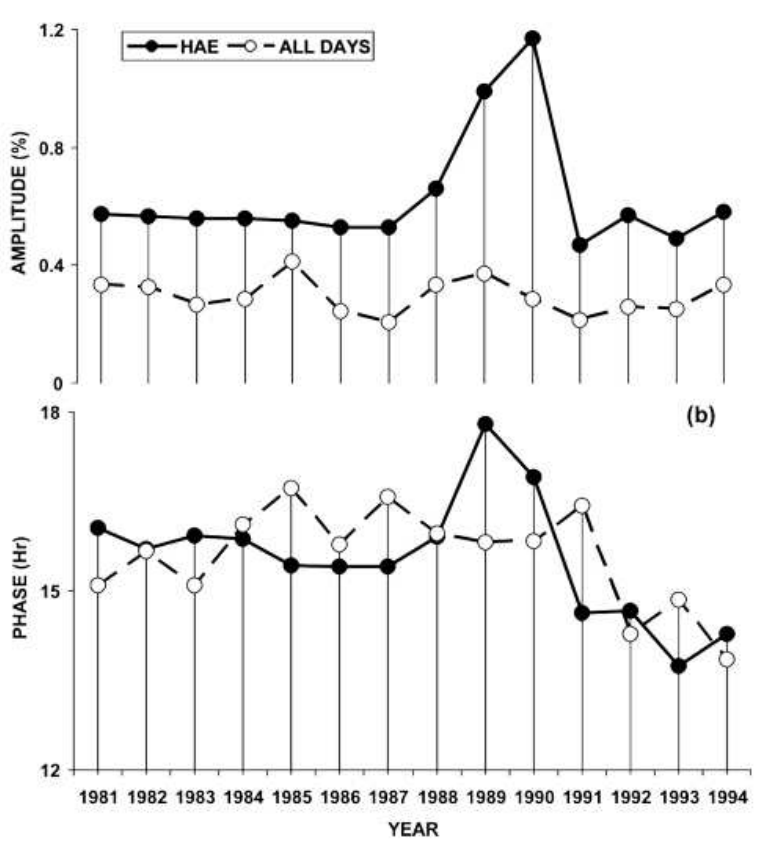

FIG. 4: Cross plots between the annual average values of the first harmonic (a) diurnal amplitude (\%), (b) diurnal time of maximum (Hr) and (c) frequency of occurrence of HAEs for Deep River NM station and the 10.7-cm solar radio flux along with the best-fit curve, R-squared value and correlation coefficient ( $r$ ) for the period 198194.

To find out a possible dependence of the amplitude, phase and occurrence of HAEs with solar activity, we have plotted the graph of amplitude, phase and frequency of occurrence of $\mathrm{HAE}$ as a function of annual average $10.7-\mathrm{cm}$ solar radio flux along with regression line, $\mathrm{R}$-squared value (The $\mathrm{R}$-squared value, also known as the coefficient of determination, is an indicator that ranges in value from 0 to 1 and reveals how closely the estimated values for the trend line correspond to your actual data. A trend line is most reliable when its R-squared value is at or near 1) and correlation coefficient (r) are shown in Fig. 4. It is clearly seen from the figure that the amplitude slightly increases and phase of diurnal anisotropy shifts towards later with the increase of $10.7-\mathrm{cm}$ solar radio flux and shows very nominal correlation with amplitude $(\mathrm{r}=0.48)$ and phase $(\mathrm{r}=0.47)$. However, it is also noteworthy from the figure that the frequency of occurrence of HAEs seems to slightly decrease with the increase of $10.7-\mathrm{cm}$ solar radio flux and shows a very weak correlation of $(r=-0.36)$. Thus, we can say the amplitude as well as phase of diurnal anisotropy shows some dependence on the annual average $10.7-\mathrm{cm}$ solar 
radio flux during the periods of HAEs, whereas, the occurrence of HAEs do not show any significant correlation with $10.7-\mathrm{cm}$ solar radio flux.

\section{CONCLUSIONS}

From the above analysis and observations we may conclude the following:

The amplitude of the diurnal anisotropy as well as time of maximum shows a good positive correlation with the solar activity cycle during high amplitude wave trains.

The diurnal time of maximum for both high amplitude days as well as for all days is found to significantly shift towards an earlier time as compared to the corotational direction since 1991.

The occurrence of high amplitude anisotropic wave train events is dominant during solar activity minimum as well as maximum years.

The diurnal amplitude as well as $10.7-\mathrm{cm}$ solar radio flux significantly deviates and reaches its maximum and phase remains in the corotational direction $(\approx 15.5 \mathrm{Hr})$ at Deep River station for majority of the HAE and shift towards later hours during solar activity maximum.

The amplitude of diurnal anisotropy and $10.7-\mathrm{cm}$ solar radio flux significantly enhanced and the phase of the diurnal anisotropy remain in the corotational during solar activity maximum years for HAEs.

The amplitude as well as phase of diurnal anisotropy shows very nominal correlation with $10.7-\mathrm{cm}$ solar radio flux $(\mathrm{r}=$ $0.48,0.47)$. However, the frequency of occurrence of HAEs shows a very weak correlation $(\mathrm{r}=-0.36)$ with $10.7-\mathrm{cm}$ solar radio flux.

\section{Acknowledgements}

The authors are indebted to various experimental groups, in particular, Prof. Margret D. Wilson, Prof. K. Nagashima, Miss. Aoi Inoue and Prof. J. H. King for providing the data.
[1] I. V. Dorman, L. I. Dorman, J. Geophys. Res. 72, 1513 (1967).

[2] K. Nagashima, I. Morishita, $16^{\text {th }}$ Int. Cosmic Ray Conf. 3, 325 (1979).

[3] J. A. Lockwood, W. R. Webber, J. Geophys. Res. 84, 120 (1979).

[4] W. R. Webber, J. A. Lockwood, J. Geophys. Res. 93, (A8) 8735 (1988).

[5] H. Mavromichalaki, E. Marmatsouri, and A. Vassilaki, Earth, Moon and Planets 42, 233 (1988).

[6] J. R. Jokipii, E. H.Levy, and W. B. Hubbard, Ap. J. 213, 861 (1977).

[7] C. J. Hatton, Solar Phys. 66, 159 (1980).

[8] H. Mavromichalaki, B. Petropoulos, Earth, Moon and Planet 37, 79 (1987).

[9] H. Mavromichalaki, A. Belehaki, and X. Rafios, Astron. Astrophys. 330, 764 (1998).

[10] Rajesh K. Mishra, Rekha Agarwal Mishra, Ind. J. Radio and Space Phys. 33, 285 (2004)

[11] S. P. Agrawal, M. Bercovitch, $18^{\text {th }}$ Int. Cosmic Ray Conf. 3, 316 (1983).

[12] H. S. Ahluwalia, Geophys. Res. Lett. 15, 287 (1988).

[13] J. J. Quenby, A. Hashim, Planetary Space Sci. 17, 1121 (1969).

[14] U. R. Rao, A. G. Ananth, and S. P. Agrawal, Planet. Space Sci. 20, 1799 (1972).
[15] A. G. Ananth, The time variation of cosmic rays intensity, $\mathrm{Ph}$. D. Thesis, Gujarat University, Ahmedabad (INDIA), (1975).

[16] M. A. Forman, L. J. Gleeson, Astrophys. Space Sci. 32, 77 (1975).

[17] E. N. Parker, Planetary Space Sci. 12, 735 (1964).

[18] W. I. Axford, Planetary Space Sci. 13, 115 (1965).

[19] S. P. Agrawal, R. L. Singh, $14^{\text {th }}$ Int. Cosmic Ray Conf. 4, 11293 (1975).

[20] R. S. Yadav, Badruddin, $18^{\text {th }}$ Int. Cosmic Ray Conf. 2, 366 (1983).

[21] H. S. Ahluwalia, J. F. Riker, $19^{\text {th }}$ Int. Cosmic Ray Conf. 5, 116 (1985).

[22] A. G. Ananth, S. P. Agrawal, and U. R. Rao, Pramana-J. Phys. 3, 74 (1974).

[23] C. M. Tiwari, D. P. Tiwari, S. P. Agrawal, and P. K. Shrivastava, Ind. J. Radio Space Phys. 33, 95 (2004).

[24] R. P. Kane, Ind. J. Radio Space Phys. 34, 299 (2005).

[25] S. Kumar, U. Gulati, and D. Khare, $21^{\text {st }}$ Int. Cosmic Ray Conf. 6, 330 (1990).

[26] A. G. Ananth, D. Venkatesan, and S. Pillai, Solar Phys. 143, 187 (1993).

[27] S. Kudo, S. Mori, $21^{\text {st }}$ Int. Cosmic Ray Conf. 6, 307 (1990). 